\title{
Cloning, purification, and functional characterization of Carocin S2, a ribonuclease bacteriocin produced by Pectobacterium carotovorum
}

\author{
Yung-Chieh Chan ${ }^{1}$, Jian-Li Wu ${ }^{1}$, Huang-Pin Wu ${ }^{2}$, Kuo-Ching Tzeng ${ }^{3}$ and Duen-Yau Chuang ${ }^{1 *}$
}

\begin{abstract}
Background: Most isolates of Pectobacterium carotovorum subsp. carotovorum (Pcc) produce bacteriocins. In this study, we have determined that Pcc strain F-rif-18 has a chromosomal gene encoding the low-molecular-weight bacteriocin, Carocin S2, and that this bacteriocin inhibits the growth of a closely related strain. Carocin S2 is inducible by ultraviolet radiation but not by mutagenic agents such as mitomycin $\mathrm{C}$.

Results: A carocin S2-defective mutant, TF1-2, was obtained by Tn5 insertional mutagenesis using F-rif-18. A 5706-bp DNA fragment was detected by Southern blotting, selected from a genomic DNA library, and cloned to the vector, pMS2KI. Two adjacent complete open reading frames within pMS2KI were sequenced, characterized, and identified as caroS2K and caroS2I, which respectively encode the killing protein and immunity protein. Notably, carocin S2 could be expressed not only in the mutant TF1-2 but also in Escherichia coli DH5 $\alpha$ after entry of the plasmid pMS2KI. Furthermore, the C-terminal domain of CaroS2K was homologous to the nuclease domains of colicin D and klebicin D. Moreover, SDS-PAGE analysis showed that the relative mass of CaroS2K was $85 \mathrm{kDa}$ and that of CaroS2l was $10 \mathrm{kDa}$.
\end{abstract}

Conclusion: This study shown that another nuclease type of bacteriocin was found in Pectobacterium carotovorum. This new type of bacteriocin, Carocin S2, has the ribonuclease activity of CaroS2K and the immunity protein activity of Caros2l.

\section{Background}

The phytopathogenic enterobacterium, Pectobacterium carotovorum subsp. carotovorum, is a phytoparasitic, Gram-negative, facultative anaerobic bacterium [1]. Pcc produces many extracellular pectic enzymes (pectate lyase, pectin lyase, exopolygalacturnoate lyase) and hydrolytic enzymes causing soft-rot disease, tissue maceration, and cell wall collapse $[2,3]$. The only current strategy against soft-rot disease involves chemical agents that unavoidably contaminate the environment [4]. Kikumoto et al. have demonstrated that mixed bacteriocin-producing avirulent strains of Pcc show high efficacy against soft-rot disease of Chinese cabbage [5].

\footnotetext{
* Correspondence: chuang@dragon.nchu.edu.tw

'Department of Chemistry, National Chung-Hsing University, 250, Kuokuang

Rd., Taichung, 402, Taiwan

Full list of author information is available at the end of the article
}

Bacteriocins are bactericidal, extracellular toxins, produced by both Gram-positive and Gram-negative bacteria [6,7]. These proteinaceous molecules kill closely related bacteria. The susceptible cell is recognized by specific target receptors on the membrane, and the producer cell evades lethality by expressing a cognate immune protein. The colicin family produced by Escherichia coli is divided into DNase (colicins E2, E7, E8 and E9), RNase (colicins E3, E4 and E6), tRNase (colicins $\mathrm{D}$ and E5), and pore-forming colicins (colicins A, E1, Ia and $\mathrm{Ib}$ ) [8]. Bacteriocins (especially nuclease bacteriocins) have a high amino acid sequence homology.

Natural bacteriocin molecules act via a number of mechanisms. For example, colicin E3 is a well-known ribonuclease that specifically cleaves $16 \mathrm{~S}$ rRNA at the 3 '-end of the coding sequence both in vivo and in vitro, which leads to the abolishment of protein synthesis 
resulting in death of the susceptible cell [9-12]. Previous reports indicate that colicin E3 consists of a killer protein with three domains (i.e., a translocation domain [ $T$ domain], receptor binding domain [ $R$ domain], and nuclease domain) and an immunity protein that retards antibiotic activity $[13,14]$. The $\mathrm{R}$ domain recognizes a specific receptor, BtuB on the cell membrane and the $\mathrm{T}$ domain interacts with the TolB protein in the cell periplasm of the sensitive cell to facilitate entry of the killer domain through the cell membrane. In addition to the attack mechanism, the immunity mechanism has been extensively elucidated. Notably the immunity protein and the killer protein interact initially at very high affinity because of charge attraction, and are separated at the cell surface through energy generated from the proton motif force [15-17].

In general, the $\mathrm{C}$-terminal domain determines the type of bacteriocin. The C-terminal nuclease domains are not only interchangeable but also lack species specificity [18]. Strikingly, the tRNase type of bacteriocin may accelerate exhaustion of tRNA in the cytoplasmic pool and thereby impair protein synthesis in vivo. Ogawa et $a l$. have demonstrated that particular tRNA molecules can be digested by colicin D as well as by colicin E5 $[19,20]$. It has been suggested that phage-associated klebicin $D$ is a tRNase type of bacteriocin based on similarity to the nuclease-like domain of colicin D [21].

Nguyen et al. reported production of a high-molecularweight bacteriocin (carotovoricin Er) and Chuang et al. reported production of a low-molecular-weight bacteriocin (LMWB; carocin) by Pectobacterium[22,23]. The former has a bulky antenna-like tail, inner core, and contractile cylindrical structure, and the carotovoricin-caused inhibition zone can be easily distinguished from that of carocin by its low diffusibility. Carocin $\mathrm{S} 1$ is a deoxyribonuclease type of LMWB (indicated by the letter S) and is secreted by Pcc strain $89-\mathrm{H}-4$. Additionally, export of Carocin S1 utilizes the type III secretion system in Pcc, which also controls the cell motility of the bacterium [24].

Pcc strain F-rif-18 is a spontaneous rifampin-resistant mutant of the wild-type 3F-3. Ultraviolet radiation can induce Pcc strain F-rif-18 to produce the LMWB Carocin S2. One of several sensitive cells, SP33, was selected as an indicator strain here. In the present study, the chromosomal bacteriocin gene, carocin $\mathrm{S} 2$, was introduced into an expression plasmid encoding two proteins, CaroS2K and CaroS2I. These proteins were purified and characterized and their primary activities of killing (CaroS2K) and immunity (CaroS2I) were investigated in vivo and in vitro.

\section{Results}

\section{Isolation of Transposon Insertion Mutants}

Conjugation between F-rif-18 and E. coli 1830 resulted in $\sim 3,500$ colonies after selection on Modified Drigalski's agar medium containing rifampin and kanamycin. In bacteriocin assay, the size of the inhibition zone around each isolate was compared with that of F-rif-18. Mutant colonies were identified by smaller inhibition zones. This evidence of mutation suggested that transposon $\operatorname{Tn} 5$ had been inserted into LMW bacteriocin-related genes. The strain TF1-2, a putative insertion mutant, would no longer produce LMW bacteriocin (Figure 1).

To ascertain whether Tn5 was actually introduced into the genomic DNA of putative isolates, the nptII gene of isolates was amplified using two primers P3 and P4 [23]. Southern blot technology showed that Tn 5 had been inserted (Additional file 1, Figure S1).

\section{Identification of Tn5-inserted DNA Structures}

To identify Tn5-interrupted genes, genomic DNA from TF1-2 was amplified with TAIL-PCR using an array of specific primers (Additional file 1, Figure S8). A 2621-bp DNA fragment, including two open reading frames (ORFs), was identified as the sequence containing the bacteriocin structural gene. This gene was designated the carocin $S 2$ gene. To characterize the carocin $S 2$ gene, the TF1-2 probe was designed to hybridize in Southern blots with a Bam HI-digested DNA fragment from the genomic library of F-rif-18 (Figure 2A). A 5706-bp Bam HI-digested DNA fragment (Figure 2B), harboring two complete ORFs of carocin S2, was cloned into the plasmid pMCL210 (Additional file 1, Figure S2). The carocin-

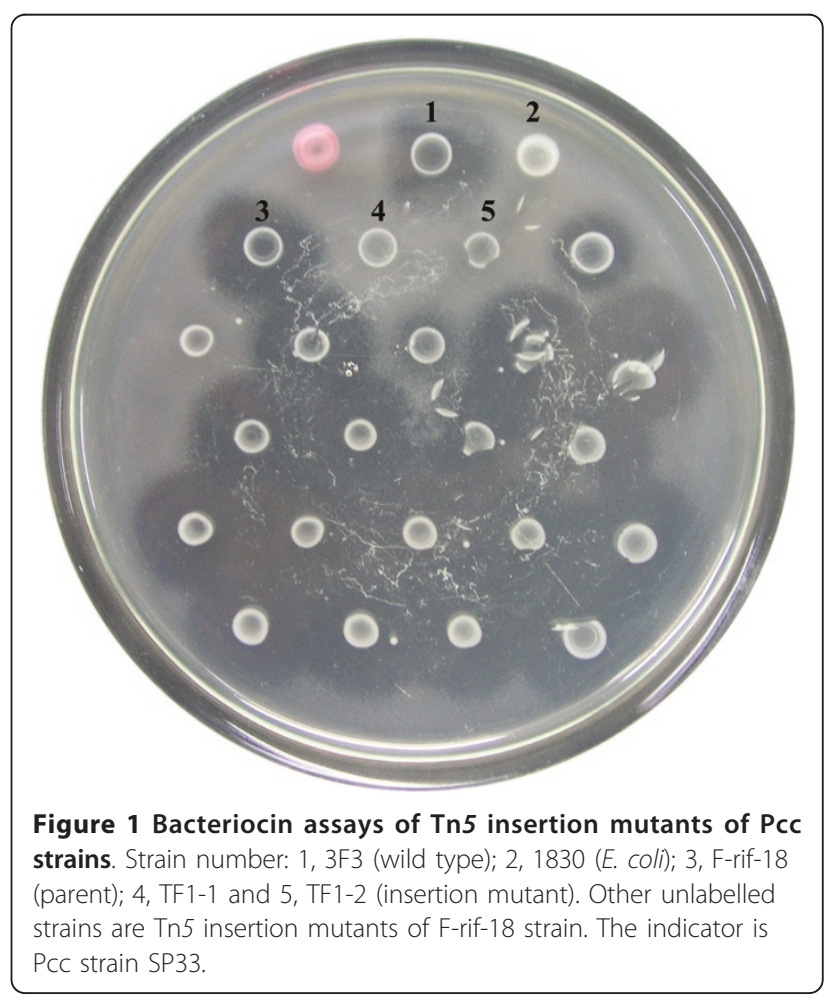



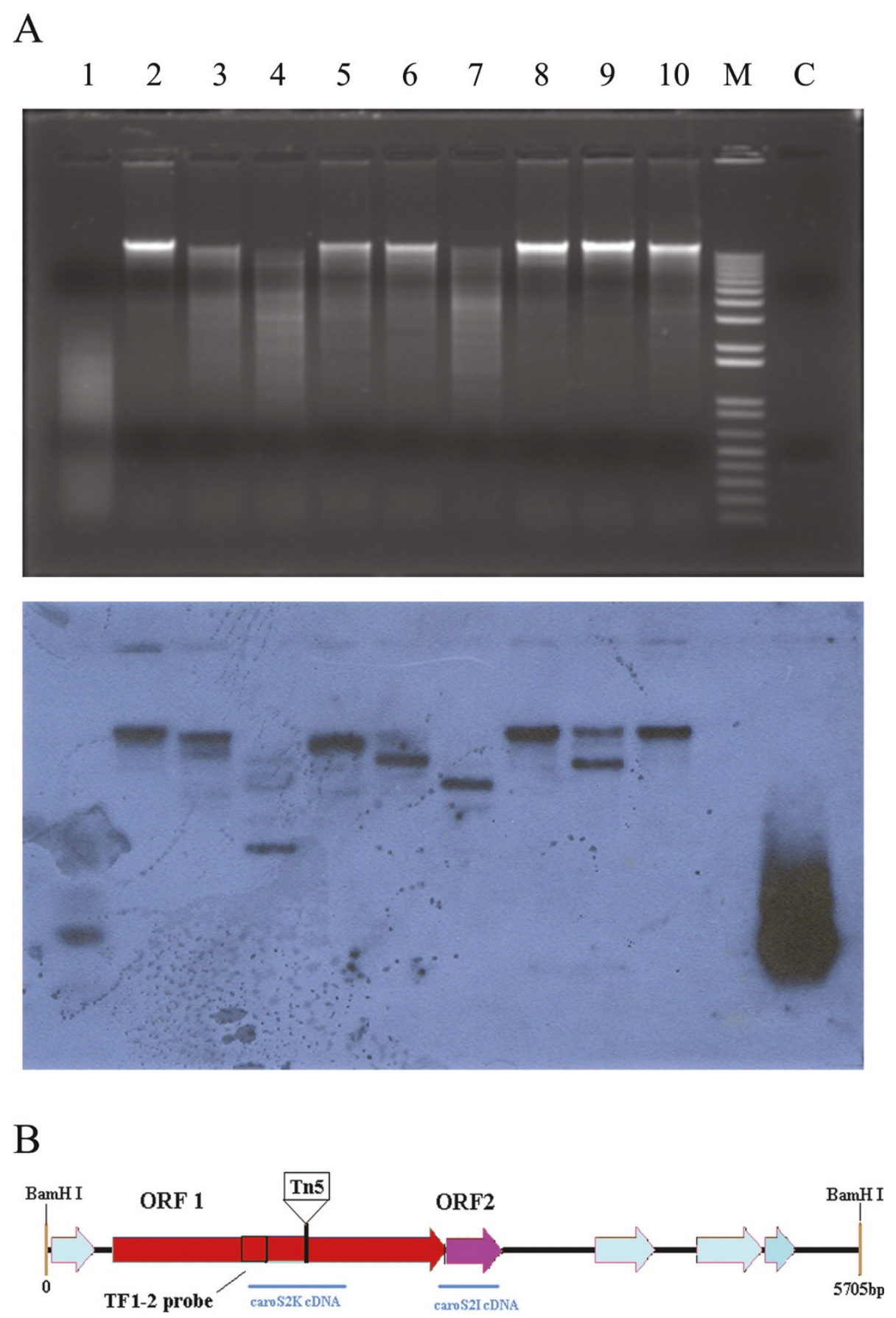

Figure 2 DNA library screening and scheme of carocin S2 gene. (A) The TF1-2 probe was used to screen DNA fragments from the genomic DNA library of F-rif-18. The DNA was digested with various restriction enzymes as follows: 1. Hpy188l; 2. Hindlll; 3 Hpal; 4. EcoRV; 5. EcoRl; 6. Clal; 7. BsaAl; 8. Bg/ll; 9. BamHl; 10. Ahdl; M. DNA leader marker; C. The TF1-2 probe DNA. The arrowhead indicates the 5.7-kb carocin S2 fragment. (B) Shown is the $5.7-\mathrm{kb}$ segment of DNA containing the carocin S2. The location of TF1-2 probe and part amplicon of cDNA of caroS2K and caroS21 were shown.

producing plasmid was designated as pMS2KI. The amplicon, comprising the predicted ORF2 of caroS2I, was subcloned into the pGEM-T easy vector, resulting in the plasmid pGS2I (Additional file 1, Figure S5).

\section{Transcriptional analysis and in vivo expression of carocin} S2 gene

To determine whether the carocin $S 2$ gene is transcribed in a series of recombinant strains, reverse transcription- 
PCR was used to estimate RNA level. Two sets of intergenic primers were designed to amplify parts of transcripts from caroS2K or caroS2I, respectively (Figure 2B). Amplification of parts of $16 \mathrm{~S}$ ribosomal RNA transcripts indicated that RNA in these bacterial cells is expressed at normal levels (Figure 3).

The presence of the 925-bp amplicon revealed that caroS $2 K$ was being transcribed in the cell (panel car$o S 2 K$ in Figure 3). The TF1-2 strain, which is a Tn5 insertional mutant, could not transcribe $\operatorname{caroS} 2 K$ (lane 2), but the ability of TF1-2 to transcribe $\operatorname{caroS} 2 K$ was restored by introduction of pMS2KI (lane 3). It was apparent that the amount of $\operatorname{caroS} 2 K$ expression was dependent on the number of copies of plasmid pMS2KI (compare lane 1 to lane 3). Additionally, carocin S2 can be expressed in $E$. coli strain $\mathrm{DH} 5 \alpha$ by introduction of pMS2KI (lane 4 and lane 5).

The presence of a 259-bp amplicon showed that caroS2I was transcribed constitutively (panel caroS2I in Figure 3). The caroS2I gene was transcribed unexpectedly in mutant strain TF1-2 even though the plasmid pMS2KI was introduced (lane 3). This demonstrated that $\operatorname{caroS} 2 I$ is expressed constitutively regardless of whether the gene $\operatorname{caros} 2 K$ is transcribed. Possibly an individual promoter for caroS2I gene is located behind the Tn5 insertion site in the $\operatorname{caroS} 2 K$ gene. CaroS2I transcripts were detected in strain SP33 with plasmid pGS2I (lanes 6 and 7). Although both the SP33 strains (with or without pGEM T-easy) were susceptible to Carocin S2, SP33/pGS2I appeared to grow in the presence of CaroS2K (Figure 4B).

To prove that pMS2KI contained the gene for Carocin S2, pMS2KI was introduced into TF1-2 and E. coli DH5 $\alpha$. Both TF1-2/pMS2KI and DH5 $\alpha /$ pMS2KI had ability to express the activity of Carocin S2 (Figure 4A). The size of inhibition zone around strain TF1-2/ pMS2KI was equal to that around DH5 $\alpha /$ pMS2KI but still smaller than that around the wild-type strain F-rif- 18. On the other hand, the quantity of transcripts expressed in vivo and in vitrodid not usually correspond.

\section{Deduction of the amino acid sequence of Carocin S2}

The carocin $S 2$ gene consists of two ORFs (Additional file 1, Figure S7): one containing the 2352-bp caroS2K gene and the other containing the 273-bp caroS2I gene. The stop codon (TGA) of caroS $2 K$ overlaps the first start codon of caroS $2 I$ by 4 -bp (ATGA). The amino sequences were deduced from the nucleotide sequence

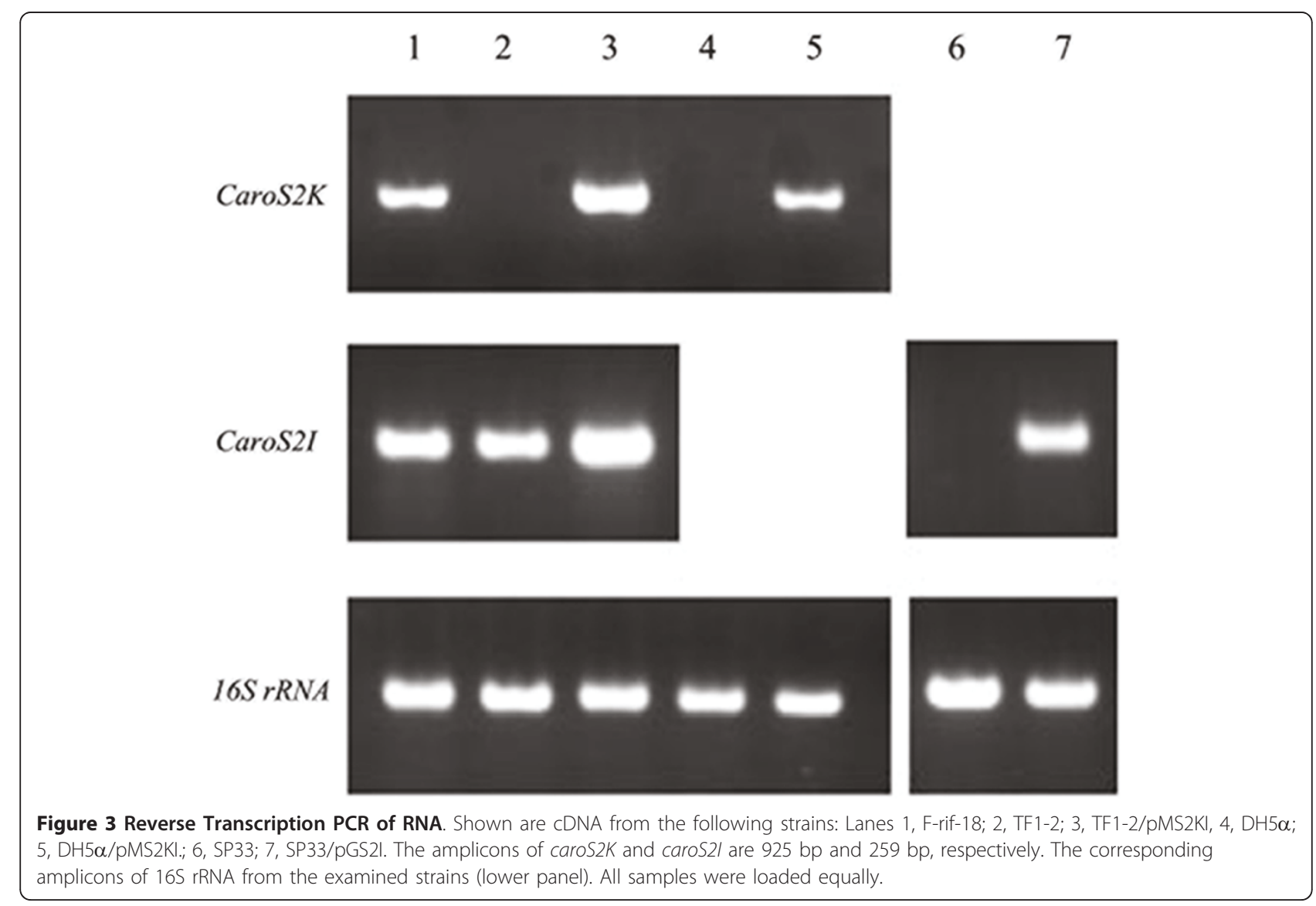




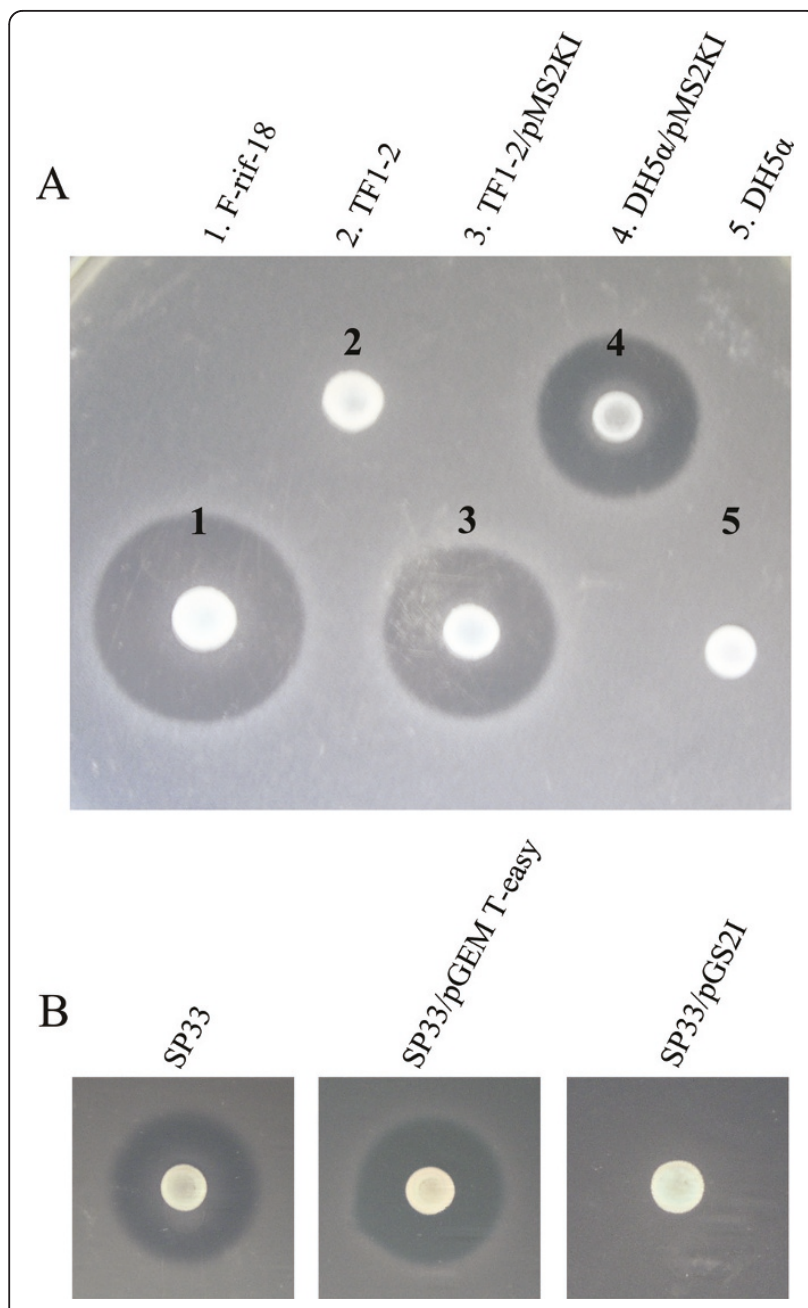

Figure 4 Recovery and immunity activity of carocin S2. (A) Antibacterial activity of carocin S2 from different strains. The indicator was PCC strain SP33. Strain number: 1, F-rif-18; 2, TF1-2; 3, TF1-2/pMS2Kl; 4, DH5 $\alpha /$ pMS2KI; 5, DH5 $\alpha$. (B) Assay for caroS2l. The colony and inoculated strains were F-rif-18. The indicator strains were: 1 , SP33; 2, SP33/pGEM-T easy; 3, SP33/pGS2I.

of the carocin $S 2$ gene using DNASIS-Mac software (HITACHI, Japan) and compared to other analogous proteins using the BLAST and FASTA search tools.

ORF1 was found to encode a 783-amino acid protein with a high degree of homology to Pcc21 carocin D, Escherichia coli colicin D and Klebsiella oxytoca klebicin D (Figure 5); ORF2 was found to encode a 90-amino acid protein that shows homology to the immunity proteins of colicin D and klebicin D (Figure 5). Thus, caroS $2 K$ produces an antibiotic with a deduced molecular mass of $85 \mathrm{kDa}$. CaroS2I (a 10-kDa protein of 90 amino acids) was shown to confer resistance to CaroS2K. It is particularly noteworthy that the homology between CaroS2K and Colicin D and Klebicin D is at the C-terminal end of these proteins where the catalytic center of a ribonuclease is located. According to the FASTA program, the amino acid segment between Asp677 and the C-terminus of CaroS2K shares almost $60 \%$ similarity with the minimal tRNase domain of colicin D and klebicin D (Figure 5). Since the colicin D and klebicin D are well-known tRNase family of bacteriocins, suggests that Carocin S2 might therefore be a ribonuclease.

\section{Purification and characterization of Carocin S2}

E. coli BL21 (DE3) recombinants, which were transformed with pES2KI or pES2I, were used to express CaroS2K protein or CaroS2I protein individually. Coomassie blue stained SDS-PAGE gels of purified Carocin $\mathrm{S} 2$ are shown in Figure 6 . The band corresponding to CaroS2K was purified. The gel indicates a relative mass $\left(\mathrm{M}_{\mathrm{r}}\right)$ of about $85 \mathrm{kDa}$ (Figure 6A), enrichment of the purified CaroS2K (arrowhead), and disappearance of other bands. Purification of CaroS2I by the same procedure resulted in a more intense band in the region of $\mathrm{M}_{\mathrm{r}} 10 \mathrm{kDa}$ (arrowhead; Figure 6B).

The purified CaroS2K involved in the growth inhibition of the susceptible indicator strain SP33 was then characterized. The number of viable cells decreased with increasing concentration of CaroS2K (Figure 7). Almost all cells were dead at the initial concentration of $4 \mu \mathrm{g}$ $\mathrm{ml}^{-1}$, indicating that about $90 \%$ of indicator strains are killed at this concentration. However, the activity of CaroS2K was inhibited by trypsin, but not inhibited by CaroS2I.

\section{Carocin S2 has ribonuclease activity}

In order to confirm the role of carocin S2 as a ribonuclease type bacteriocin, we set up a RNA degradation assay. Northern blots of $5{ }^{\prime}-{ }^{32} \mathrm{P}$-labeled total RNA extract treated with increasing concentrations of CaroS2K (Figure 8B) showed a markedly lower intensity of labeled RNA fragments compared to untreated extracted RNA (Figure 8B, lane 1), suggesting that CaroS2K has ribonuclease activity.

Surprisingly the RNA segments were larger when the RNA was $3^{\prime}-{ }^{32} \mathrm{P}$-labeled compared with $5{ }^{\prime}-{ }^{32} \mathrm{P}$-labeling (Figures $8 \mathrm{~B}$ and $8 \mathrm{C}$ ). As the concentrations of $23 \mathrm{~S}$ RNA and 16S RNA decrease on the addition of increasing concentrations of CaroS2K, it is assumed that more ribosomal RNA is degraded leaving material that is ostensibly the ribosome. When excess concentrations of caroS2K (i.e $1 \mu \mathrm{g}$ ) are added then most of the ribosomal RNA is degraded leading to a destabilization and subsequent degradation of the ribosome (Figure $8 \mathrm{C}$, lane 2). We hence consider that CaroS2K (in sufficient amount) would degrade the ribosome. CaroS2I inhibits the killing activity of CaroS2K because a mixture of equal quantities of CaroS2K and CaroS2I prevented digestion of RNA segments by CaroS2K (Figure 8C, lane 6). 


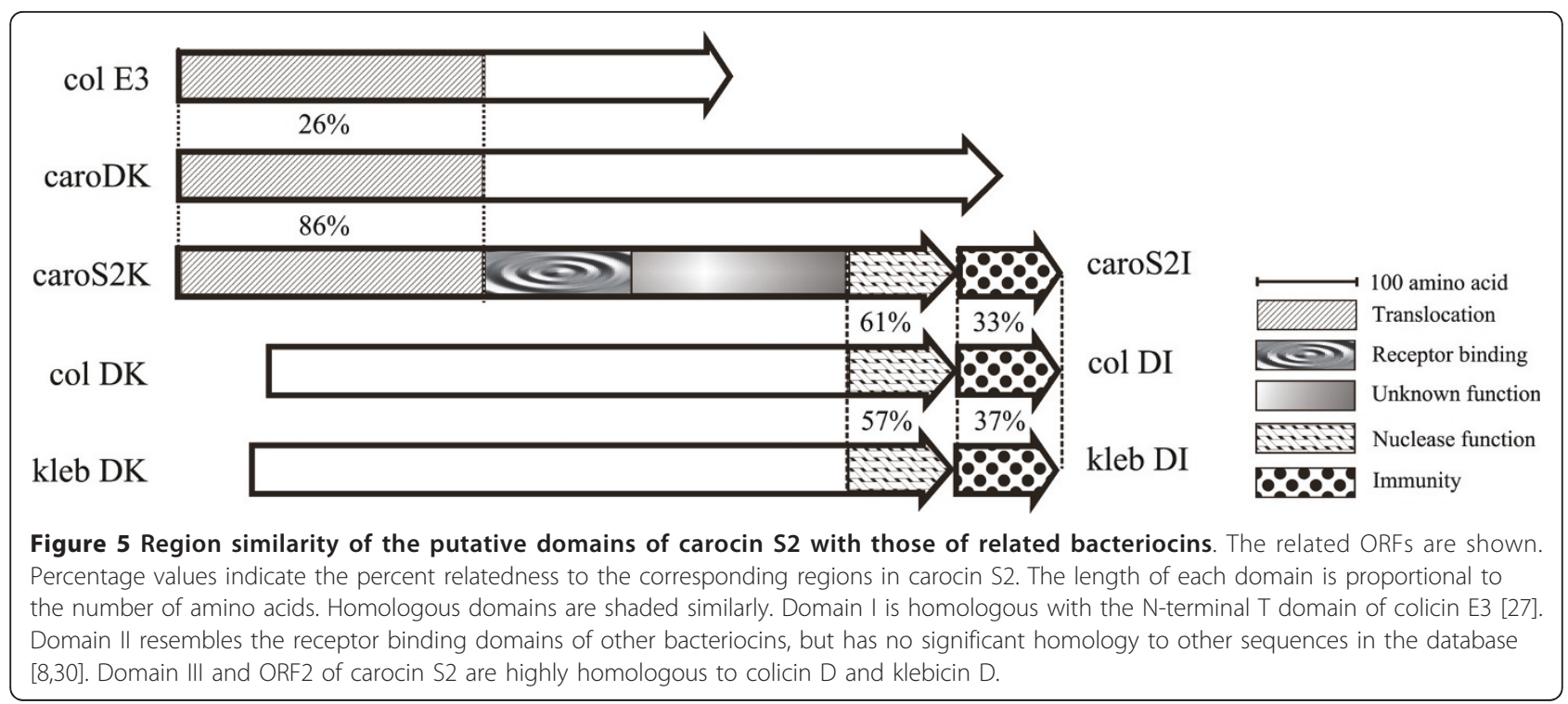

Subsequently, treatment of the genomic DNA of the indicator strain SP33 with the purified CaroS2K protein had no effect on deoxyribonuclease activity, as compared to the pattern of EcoRI-digested genomic DNA (Figure 8A and Additional file 1, Figure S4).

\section{Nucleotide sequence accession number}

The Genbank accession number of the sequence of the carocin S2 gene is HM475143.

\section{Discussion}

In this study, a chromosome-borne gene encoding bacteriocin, carocin S2, in Pcc strain 3F3 was shown to possess ribonuclease activity. According to Bradley's classification, Carocin S2 is a low-molecular-weight bacteriocin [25]. Two genes, caroS2K and caroS2I, encode the $85-\mathrm{kDa}$ and $10-\mathrm{kDa}$ components, respectively, of Carocin S2. The substrate and gene structure of carocin S2 were unlike those of other bacteriocins from Pcc.

On the basis of sequence analysis, carocin $S 2$ comprises these two overlapping ORFs, caroS2 $K$ and caroS2I (Additional file 1, Figure S7). A putative Shine-Dalgarno sequence 5'-AUGGA-3', which has also been seen in the DNA sequence of carocin S1, is located upstream (-9 bp to $-13 \mathrm{bp}$ ) of the start codon AUG, suggesting that it could be a ribosome binding site for caroS2K [23]. Comparison of the upstream sequences of both $\operatorname{caroS} 2 \mathrm{~K}$ and caroS2I has shown that the two consensus sequences, 5'-TATAAAAA-3' (-34 bp to $-41 \mathrm{bp}$ ) and 5'-GAAGT-3' ( $-61 \mathrm{bp}$ to $-65 \mathrm{bp})$, are both upstream from the start codon. Presumably, 5'-TATAAAAA- 3 ' is the -10 promoter and 5'-GAAGT-3' is the -35 promoter for the

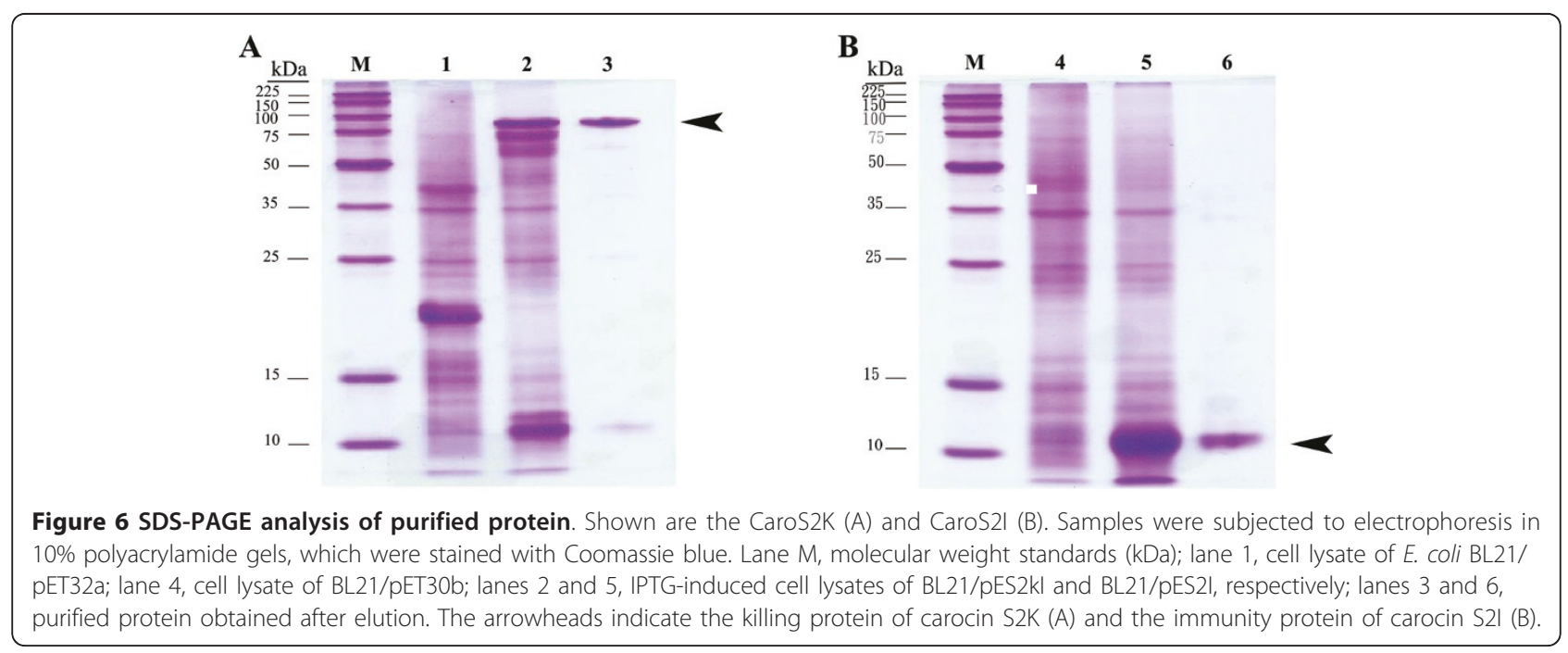




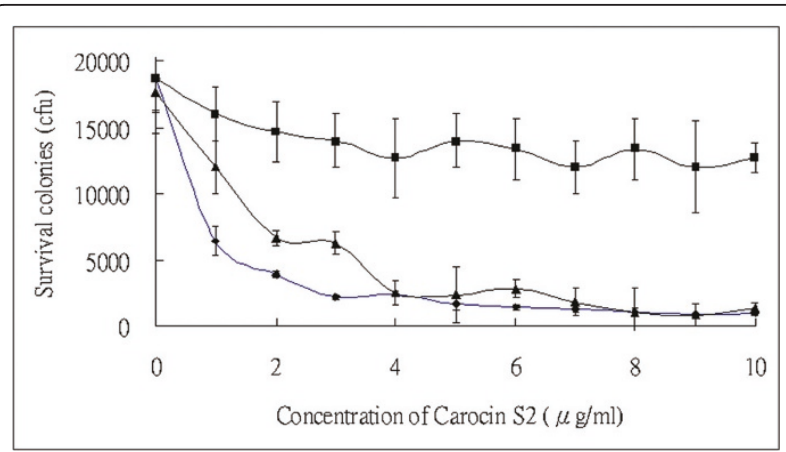

Figure 7 Survival of SP33 cells treated with Carocin S2. Aliquots of indicator SP33 cells were treated with increasing concentrations of CaroS2K $(\bullet)$ and CaroS2K:CaroS2I in molar ratio of 1:1 ( $\mathbf{(})$. The effect of trypsin on the Caros2K was also assayed (-). The data are reported as means \pm standard deviations.

carocin S2 gene, even though they differ from those of E. coli[26].

A putative -10 promoter is 33 bp upstream from the initiator ATG of the caroS $2 K$ gene, in which the SD sequence is embedded, while the -35 promoter is $19 \mathrm{bp}$ upstream of the -10 promoter region. The putative promoter of the -35 box of caroS2I is located similarly near the -10 box, but the -10 box is just 24 bp upstream of the start codon where no SD sequence is apparent. Although those hypothesized promoters are located within the $\operatorname{caroS} 2 K$ structural gene, transcripts of car$o S 2 I$ are routinely produced (Figure 3 ). This suggests that caroS2I RNA expression may be regulated posttranscriptionally, in spite of close neighboring genes downstream of the gene $\operatorname{caroS} 2 K$; that is, core promoter elements may influence the expression of caroS2I gene.

In the present study, we attempted to separate CaroS2K from CaroS2I attached to (His) ${ }_{6}$-tag using a Nickel column (pEH2KI; Additional file 1, Figure S5), but a small amount of CaroS2I $(\mathrm{Mr} \sim 10 \mathrm{kDa})$ was observed in SDS-PAGE gels (Figure 6, bottom in lane 3), which had little influence on the activity of CaroS2K as the purified protein still had transient killing activity. Additionally, the activity of the Carocin S2 complex at $4^{\circ} \mathrm{C}$ was longlasting indicating good stability.

The C-terminal amino acid sequence of Carocin S2 had higher homology to those of colicin D and klebicin D, which are produced by E. coli and Klebsiella oxytoca, respectively, than to the amino acid sequence of carocin S1 from the same species (Additional file 1, Figure S6B).

The amino acid sequence of CaroS2K has three putative domains. Domain I (the N-terminal 314-residue sequence ending in Pro314) is regarded as the translocation domain and is homologous to the translocation domains of carocin D and colicin E3 (Figure 5). It is assumed to direct the cytotoxic domain to the periplasmic space $[27,28]$. Additionally, the putative TonB box (a sequence recognition motif DTMTV) was found in the N-terminal domain of CarocinS2, which is thought to participate in bacteriocin translocation [8]. Thus, we suggested that Carocin S2 could be a TonB-dependent bacteriocin.

Domain III (extending from Asp677 to the carboxyl terminus) is the killer domain. Particularly noteworthy is the resemblance of the killer domain to the tRNase domain of colicin D and klebicin D (Figure 5), and thus we suggested that carocin S2 might have tRNase activity [29-31]. Domain II extends 141 residues from Ilu315 to Val455 and is hypothesized to be the binding site that recognizes specific receptors on cell membranes. Additionally, domain III has no significant homology to carocin D, suggesting that carocin S2 and carocin D have different functions [28].

Finally, we showed that total RNA (whether labeled with radioactive phosphate at the 5'- or at the 3'-end) is sensitive to Carocin S2. Carocin S2 degraded 5'labeled total RNA but not 5'-labeled CaroS2K-free RNA (Figure 8B), and the amount of degradation was not dose-dependent (arrowhead). However, the appearance of segments of unknown origin paralleled partial degradation of $23 \mathrm{~S}$ and $16 \mathrm{~S}$ rRNA (Figure $8 \mathrm{C}$ ). These results suggest that the site of excision (either conformational or sequential) is close to the 5'-terminus of rRNA. Notably, the decrease in the amount of rRNA depended on the amount of Carocin S2 protein present, with complete degradation occurring in the presence of excess Carocin S2. Ogawa et al. reported that RNase type of bacteriocins, colicin E3 and colicin E5, catalyze the hydrolysis of the shorter RNAs from 16S rRNA [19,32]. Moreover, colicin E5 was found to hydrolyze tRNA in vitro. Furthermore, it was previously reported that colicin E3 cleaved 16S rRNA completely, and even $30 \mathrm{~S}$ rRNA [11,33]. In our study, carocin $\mathrm{S} 2$ acted as an RNase that hydrolyzes rRNA (both 23S and 16S) in vitro. In terms of enzymatic function, Carocin S2 may act as an endo- and exo-ribonuclease simultaneously. Moreover, CaroS2I significantly inhibited nuclease activity in vitro but not in vivo (Figures 7, Figure 8 andAdditional file 1, Figure S3). We speculated that immunity protein CaroS2I might not be able to cross the cell membrane, as previously described [14]. Although our in vitro experiment showed that carocin S2 was a ribonuclease, further investigation is needed to clarify its function in cells.

One of the other Tn5 insertional mutants, TF1-1, which disrupted the coding sequence of the $\mathrm{fliC}$ gene, was found to halt expression of Carocin S2 (Figure 1), indicating that Carocin S2 can also be secreted via the type III secretion system [24]. The role of carocin S2 as an RNase in the cytoplasm is to prevent protein synthesis by cleaving either $23 \mathrm{~S}$ rRNA or $16 \mathrm{~S}$ rRNA. The role 


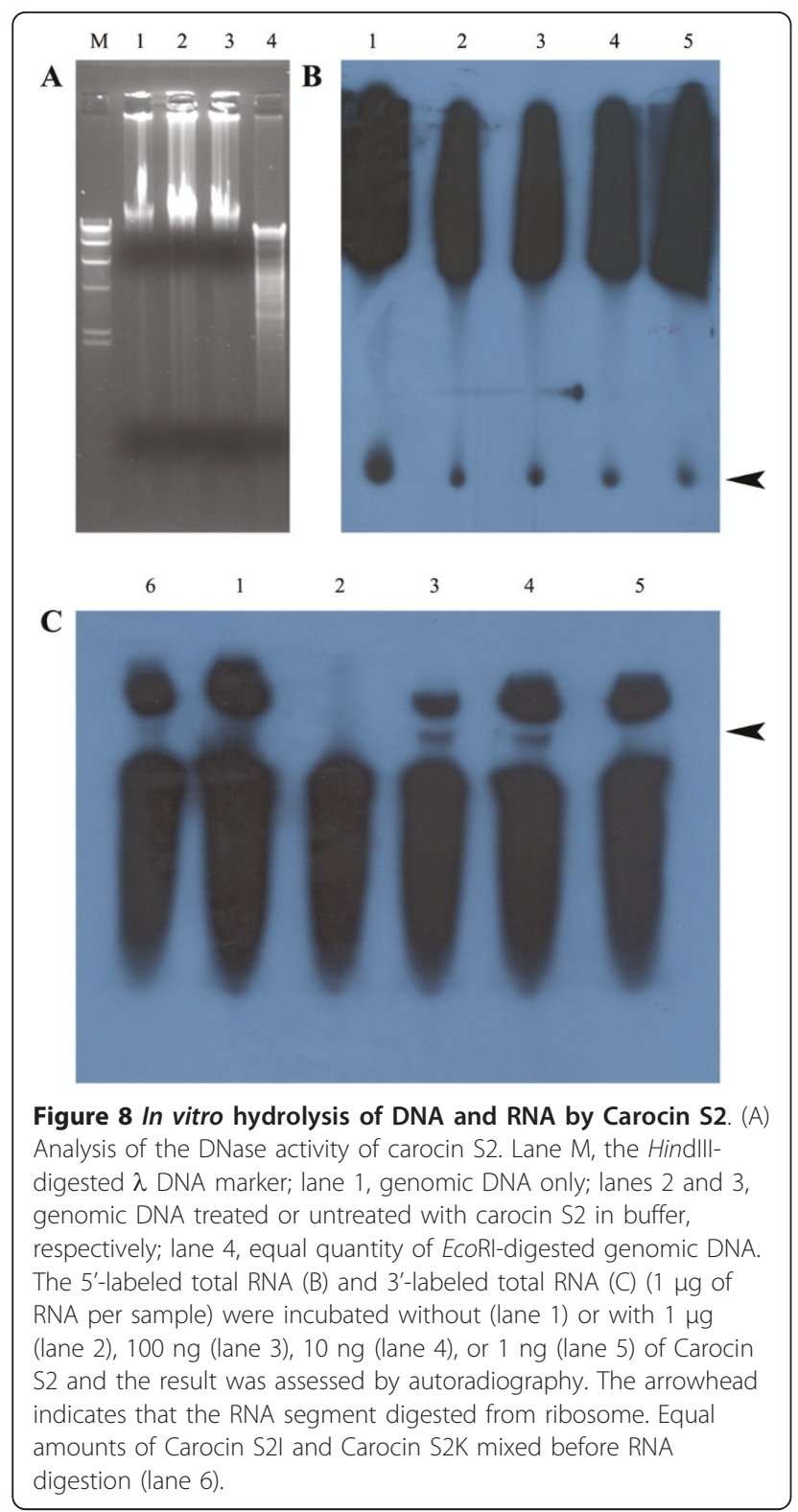

of the immunity protein, CaroS2I, is usually to stop the damage caused by CaroS2K in the cytoplasm. More details of the actual mechanism of carocin S2 remain to be elucidated.

\section{Conclusion}

As shown herein, the novel bacteriocin, Carocin S2, was characterized as a ribonuclease. It is the first bacteriocin with ribonuclease activity to be found in Pectobacterium strains. We suggested that Carocin S2 kills the indicator cell by exhausting its supply of some kinds of RNA, leading to inactivation of protein biosynthesis. It will be of interest to study the proteomics of Carocin S2 and its mechanism of action in the future.

\section{Methods}

Bacterial strains, media, and growth conditions

Bacterial strains and plasmids used in the study are listed in Table 1. Isolates of Pcc were grown at $28^{\circ} \mathrm{C}$ in Luria-Bertani (LB) medium or IFO-802 medium. The IFO-802 medium was supplemented with $1 \%$ polypeptin, $0.2 \%$ yeast extract, $0.1 \% \mathrm{MgSO}_{4}(\mathrm{pH} 7.0)$, and $1.5 \%$ agar. Isolates of Pcc were distinguished from Escherichia coli by their ability to grow on Modified Drigalski's agar

\section{Table 1 Bacteria and plasmids used in the study}

\begin{tabular}{|c|c|c|}
\hline Strain or plasmid & Description & Source \\
\hline \multicolumn{3}{|l|}{ Escherichia coli } \\
\hline 1830 & $\begin{array}{l}\text { pro }^{-} \text {met }^{-} \mathrm{Kan}^{r} \mathrm{Nm}^{\mathrm{r}} \text {, containing } \\
\text { transposon Tn5 on the suicidal } \\
\text { plasmid pBJ4JI }\end{array}$ & [44] \\
\hline $\mathrm{DH} 5 \alpha$ & 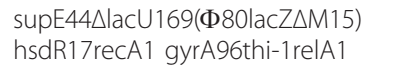 & {$[39]$} \\
\hline BL21(DE3) & $\begin{array}{l}\text { hsdS gal( } \lambda \text { clts857 ind } 1 \text { Sam7 nin5 } \\
\text { lac UV5-T7 gene 1) }\end{array}$ & {$[45]$} \\
\hline \multicolumn{3}{|l|}{$\begin{array}{l}\text { Pectobacterium } \\
\text { carotovorum subsp. } \\
\text { carotovorum }\end{array}$} \\
\hline $3 F-3$ & Pcc, wild-type & $\begin{array}{l}\text { Laboratory } \\
\text { stock }\end{array}$ \\
\hline F-rif-18 & 3F3, Rif', wild-type & This study \\
\hline TF1-1 & F-rif-18, flic::Tn5, Rif', Kan' & This study \\
\hline TF $1-2$ & F-rif-18, CarocinS2::Tn5, Rif', Kan'r & This study \\
\hline SP33 & Pcc, wild-type & $\begin{array}{l}\text { Laboratory } \\
\text { stock }\end{array}$ \\
\hline \multicolumn{3}{|l|}{ Plasmid } \\
\hline pMCL210 & p15A, Cml', Low copy number & {$[46]$} \\
\hline pGEM T-Easy & Ampr; lacZ cloning vector & Promega \\
\hline pET32a & $\begin{array}{l}\text { Ampr; expression vector with the } \\
\text { N-terminal His-tag }\end{array}$ & Novagen \\
\hline pET30b & $\begin{array}{l}\text { Kan'; expression vector with the } \\
\text { C-terminal His-tag }\end{array}$ & Novagen \\
\hline pMS2KI & $\begin{array}{l}\text { 5.7-kb BamHI DNA fragment } \\
\text { harboring carocin S2 gene from } \\
\text { 3F3 genome, cloned into } \\
\text { pMCL210 }\end{array}$ & This study \\
\hline $\mathrm{pEN} 2 \mathrm{~K}^{*}$ & caroS2K subcloned into pET32a & This study \\
\hline $\mathrm{pES} 2 \mathrm{KI}$ & $\begin{array}{l}\text { Derived from pEN2K; deleted } \\
\text { series of Tag element in front of } \\
\text { expressed caros } 2 K\end{array}$ & This study \\
\hline$\left.\mathrm{pEH} 2 \mathrm{~K}\right|^{*}$ & $\begin{array}{l}\text { Derived from pES2Kl; adding } \\
\text { (His) })_{6} \text { Tag adjacent to caros2l }\end{array}$ & This study \\
\hline pGS21 & $\begin{array}{l}\text { caroS2l and its putative promoter } \\
\text { from pMS2Kl, subcloned into } \\
\text { pGEM T-easy }\end{array}$ & This study \\
\hline pECS2 $1^{*}$ & $\begin{array}{l}\text { caroS2l subcloned into pET30b, } \\
\text { but the expressed fusion CaroS2I } \\
\text { has no activity }\end{array}$ & This study \\
\hline pES2l & $\begin{array}{l}\text { Derived form pECS2I, the }\left(\mathrm{His}_{\sigma^{-}}\right. \\
\text {Tag element was deleted }\end{array}$ & This study \\
\hline
\end{tabular}

Kan $^{r}$ : Kanamycin; Cml': Chloramphenicol; Rif': Rifampicin; Ampr: Ampicillin. *: See Additional file 1, Figure S5. 
medium [34]. Antibiotics (final concentration, $100 \mu \mathrm{g}$ $\mathrm{ml}^{-1}$ of media) were added when necessary.

\section{Bacterial conjugation}

Overnight cultures of Pcc (recipient) and E. coli (donor) were mixed and spread onto $0.22-\mu \mathrm{m}$ membrane filters placed on LB agar media and incubated overnight at $28^{\circ} \mathrm{C}$ [23]. The progeny after conjugation were appropriately diluted and cultivated on Modified Drigalski's medium (with ampicillin and kanamycin $\left[100 \mu \mathrm{g} \mathrm{ml}^{-1}\right]$ ) overnight at $28^{\circ} \mathrm{C}$. All isolates were placed on IFO-802 medium and tested for bacteriocins. Bacteriocin was assayed using the double-layer method, and Pcc SP33 was used as indicator strain [35]. The cells were incubated for 12 hours to form colonies, exposed to ultraviolet irradiation, incubated again for 12 hours, treated with chloroform to kill the cells, and then covered with soft agar containing indicator cells. The bacteriocin production was indicated by a zone of inhibition of indicator-cell (SP33) growth around the colony.

\section{Genetic-engineering technique}

The procedures of plasmid preparation, genomic DNA isolation, and DNA manipulation were performed as described by Sambrook et al. [36]. Oligonucleotide DNA primers were synthesized by MD Bio Inc. (Taipei, Taiwan). The PCR was amplified with Go-Taq DNA polymerase (Promega, USA). The thermal asymmetric interlaced PCR (TAIL-PCR) was performed as previously described [37].

Plasmids were introduced into Pcc strains using electroporation $(1.25 \mathrm{kV} / \mathrm{cm}, 200 \Omega, 25 \mu \mathrm{F})$ [38]. For heatshock transformation, the competent cells of $E$. coli were prepared according to the method of Hanahan [39].

Exponentially growing cells $\left(\mathrm{OD}_{595}\right.$ of about 6.0) were harvested for RNA preparation. Total RNA was isolated using Trizol reagent (Invitrogen, USA) according to the manufacturer's instructions. RNA was resuspended in diethylpyrocarbonate (DEPC)-treated water. The concentration of RNA was determined by $\mathrm{OD}_{260}$ absorption, and RNA was analyzed by electrophoresis on $1.5 \%$ formaldehyde-morpholinepropanesulfonic-agarose gel.

Reverse transcription-PCR (RT-PCR) was carried out with AMV Reverse Transcriptase (Promega Inc., Taiwan) according to manufacturer's instructions. RNA $(1 \mu \mathrm{g})$ was subjected to RT-PCR containing CaroS2_re_1 used as a reverse primer in first-strand cDNA synthesis. The RT mixtures were diluted and used as templates in a PCR reaction with two primers CaroS2_re_1 and CaroS2_for_1 (Additional file 1, Table S1).

A 2621-bp BamHI-HindIII digested DNA fragment, including the caroS2K and caroS2I genes, was amplified from pMS2KI with primers of CarocinS2K_for2 and CarocinS2I_rev2 (Additional file 1, Table S1) and subcloned into pET32a to give the plasmid pEN2K (Additional file 1, Figure S5). The pES2KI was obtained by excision of the Tag element between the rbs (ribosome binding site) and start code (for CaroS2K) in pEN2K using the SLIM method as previously described $[40,41]$. The 5IHT32a2KI_forT, 5IHTGT2KI_forS, 5IHT32a3KI_revT, and 5IHT32a4KI_revS primers were used. A 273-bp fragment of the caroS2I gene was amplified by PCR and ligated into the NdeI and XhoI site of pET30b to form the plasmid pEC2I. Similarly, the plasmid pES2I was obtained by deleting the (His) ${ }_{6}$-tag of pEC2I (carried out as described above with primers of X21_forT, X21_forS, X21_revT and X21_revS). Subsequently, pES2KI and pES2I were introduced into $E$. coli BL21 (DE3) cells, respectively.

\section{Restriction DNA library screening and Southern blots}

Southern blots were performed according to the DIG Application Manual (Roche, USA). A 543-bp DNA fragment (TF1-2 probe) was amplified with TF1-2P and TF1-2A2 primers (Additional file 1, Table S1), subcloned into pGEM-T Easy vector (Promega Inc., USA), and labeled using a Random Primed DNA Labeling Kit (Roche Diagnostics, USA).

The genomic DNA of the wild-type strain F-rif-18 was digested with various restriction endonucleases, with sites located outside the putative open reading frame. Samples were electrophoresed and analyzed with Southern blotting. After detection using the TF1-2 probe, the DNA from positive gel slices was purified and cloned into pMCL210 to give the carocin-producing plasmid pMS2KI. The pMS2KI construct was isolated and detected as above with the TF1-2 probe.

\section{Protein purification}

The transformant cells of BL21, harboring pES2KI or pES2I, were grown in $500 \mathrm{ml}$ to an $\mathrm{OD}_{595}$ of 0.4 . The cells were induced with isopropyl- $\beta$-D-thiogalactopyranoside (IPTG; final concentration, $0.1 \mathrm{mM}$; at $25^{\circ} \mathrm{C}$ for 12 h). Subsequently, the cells were pelleted and the pellets were sonicated (10 cycles of $9 \mathrm{~s}$ with 9 -s intervals). BL21/ pES2KI pellets were subjected to ammonium sulfate precipitation (30-40\%), resuspended in buffer A $(30 \mathrm{mM} \mathrm{NaCl}$ and $20 \mathrm{mM}$ Tris-Cl, pH 8.0), and applied to a Fractogel column (Merck, USA). The fraction was eluted by a $\mathrm{NaCl}$ gradient (30 mM-1.4 M). After purification through a P-100 size-exclusion column (BioRad, USA), the CaroS2K fractions were pooled and concentrated using an Amicon centriprep-50 column (Millipore, USA) and dissolved in buffer A. BL21/pES2I pellets were precipitated by ammonium sulfate (70-100\%) and resuspended in buffer A. CaroS2I purification involved a similar 
chromatographic procedure using the Amicon centriprep3 column (Millipore, USA). The concentration of protein was determined by the Bradford assay (Amresco, USA).

\section{In vitro determination of Carocin S2 activity}

Total RNA was treated with calf intestinal alkaline phosphatase (Promega, USA) at $55^{\circ} \mathrm{C}$ for $30 \mathrm{~min}$ as recommended by the manufacturer. The reaction was arrested by adding $5 \mathrm{mM}$ nitrilotriacetic acid, and RNA was extracted with equal volumes of phenol/chloroform. An aliquot of phosphatase-treated RNA was $5^{\prime}-{ }^{32} \mathrm{P}$-labeled at $37^{\circ} \mathrm{C}$ for $30 \mathrm{~min}$ by incubation with a mixture of $\left[\gamma_{-}{ }^{32} \mathrm{P}\right]$ ATP, T4 polynucleotide kinase (Promega Inc, USA), and reaction buffer in nuclease-free water [42]. $\left[5^{3}{ }^{32} \mathrm{P}\right]$ Cytidine 3',5'-bisphosphate $(\mathrm{pCp})$ and T4 RNA ligase (Promega, USA) were used for 3'-labeling of RNA [43]. Subsequently, the mixture was purified by MicroSpin G-25 columns (GE Healthcare, USA). The purified labeled RNA was divided into aliquots and incubated without or with Carocin S2 at $28^{\circ} \mathrm{C}$ for $60 \mathrm{~min}$, respectively. To measure its activity, CaroS2I was pre-mixed with an equal amount of CaroS2K. The mixtures were subjected to electrophoresis on a $9 \%$ polyacrylamide gel (19:1) containing $7 \mathrm{M}$ urea, $50 \mathrm{mM}$ Tris, $50 \mathrm{mM}$ boric acid, and $1 \mathrm{mM}$ EDTA, $\mathrm{pH}$ 8.3. All samples were electrophoresed at $15^{\circ} \mathrm{C}$ by PROTEIN II xi (BioRad, USA).

To confirm DNase activity, $1 \mu \mathrm{g}$ of genomic DNA from SP33 in solution containing buffer A was incubated with or without Carocin $\mathrm{S} 2$ at $28^{\circ} \mathrm{C}$ for $90 \mathrm{~min}$. An equal quantity of genomic DNA was digested with EcoRI at $28^{\circ} \mathrm{C}$ for $90 \mathrm{~min}$. Samples were then subjected to electrophoresis on $1 \%$ agarose gel.

\section{Antibiotic activity of Carocin S2}

Overnight cultures of SP33 were diluted (1:100) with LB medium and grown at $28^{\circ} \mathrm{C}$ to a density of approxi-

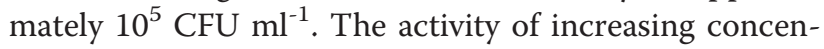
trations of Carocin S2 on cells in suspension incubated at $28^{\circ} \mathrm{C}$ for $60 \mathrm{~min}$ was assessed. CaroS2I was pre-mixed with an equal molar ratio of CaroS2K. All reaction mixtures were spread onto LB agar plates and incubated at $28^{\circ} \mathrm{C}$ for $16 \mathrm{~h}$. The experiment was performed three times. Colonies growing on a series of plates were respectively counted.

\section{Computer analysis of sequence data}

Sequencing of the DNA fragments was carried out using an ABI automated DNA sequencer 373S. The nucleotide sequence data were compiled by DNASIS-Mac software (Hitachi, Japan). Amino acid sequences were compared using international BLAST and FASTA servers. Also, the putative domains of Carocin S2 were predicted using the PSI/PHI-BLAST.

\section{Additional material}

Additional file 1: Figure S1. Analysis of Tn5 insertional mutants by southern blotting. Lane $M$, the Hindlll-digested $\lambda$ DNA marker; the genomic DNA of strains were loading as follows: lane 1, TF1-2; lane 2, Frif-18; lane 3, 3F3; lane 4, TF1-1. Lane 5, the construct pGnptll that contain the detect probe DNA nptll. The result shows that TF1-2 and TF1-1 was a Tn5 insertional mutant. Figure S2. The construct pMS2KI was cloned from genomic DNA library and screening by southern blotting with TF1-2 probe. By southern blotting, it showed that the carocin $\mathrm{S} 2$ has been cloned to form pMS2KI. Figure S3. The total RNA of SP33 were digested with Carocin S2 and electrophoresis as follows: lane 1, RNA (1 $\mu \mathrm{g})$; lane 2, RNA and CaroS2K $(20 \mu \mathrm{g})$; lane 3, RNA and Caros2l $(4 \mu \mathrm{g})$; lanes 4 to 6 are RNA $(1 \mu \mathrm{g})$ and CaroS2K $(20 \mu \mathrm{g})$ with gradient concentration of Caros2l, which were added with $4 \mu \mathrm{g}$ (lane 4); $20 \mu \mathrm{g}$ (lane 5); $100 \mu \mathrm{g}$ (lane 6). All reactions were performed at $28^{\circ} \mathrm{C}$ for 3 hours. Figure S4. Metal effect of In vitro hydrolysis of DNA by Carocin S2. Lane M, the Hindll-digested $\lambda$ DNA marker; lane 1, the genomic DNA of SP33 only; lane 2, the EcoRI-digested genomic DNA; the genomic DNA was incubated with Carocin S2 (lane 3 to 5), or not.

Magnesium acetate, nickel acetate and zinc acetate was added in buffer $\mathrm{A}(\mathrm{pH}=7)$, respectively. The reactions were performed at performed at $28^{\circ} \mathrm{C}$ for 1 hour. Figure S5. Schematic representation of the cloning strategy used in this study. (1) A 543-bp amplicon was cloned into the vector PTF1 to form the PTF1-2-probe. (2) The TF1-2 probe was prepared. (3) The multi-enzyme-digested DNA fragments were obtained from F-rif-18 genomic DNA, and they were detected on southern blots. (4) Positive CDNA was cloned into the carocin-producing plasmid pMS2KI. (5) A 2621-bp amplicon, from pMS2Kl, was subcloned into pET32a to form pEN2K. (6) The 5'-transcriptional element, which would be translated into the Flag tag, was deleted from pEN2K using the SLIM method [40]. (7) By using SLIM method, an element encoding a stretch of six histidines was inserted into caroS2l to form pEH2KI. (8) A 484-bp amplicon was subcloned into pGEM T-easy vector to form pGS2I. (9) A273-bp fragment of the caroS2l gene was amplified from pGS2I and subcloned into pET30b to form pECS2I. (10) The 3'-transcriptional element, which would be translated to $\left(\mathrm{His}_{6}\right.$-Flag, was deleted from pES2l using the SLIM method. Figure S6. Alignment of the deduced amino acid sequences of carocin S2 with those of homologous domains of bacteriocins. The potential TonB-binding motif is shown by red underline. (A) The N-terminal translocation domain of CaroS2K (Met1 to Pro314) has homology to carocin D and colicin E3. (B) The killing domain of CaroS2K (Asp677 to carboxyl terminus) has homology to the minimal tRNase domain of colicin D and klebicin D. (C) The deduced amino acid of immunity protein of CaroS2l has homology to colicin D and klebicin D. Figure S7. The gene and deduced amino acid sequence of carocin S2 shows in the study. The sequence was truncated form pMS2KI. The underline shows the putative promoter. Figure S8. Schematic representation of thermal asymmetric interlaced PCR (TAIL-PCR) were manipulated according to the method of Liu and Whittier, but the annealing temperature was decreased from $63^{\circ} \mathrm{C}$ to $60^{\circ} \mathrm{C}$ for specific primers $[37,23]$. Amplifying the unknown DNA fragment are the specific primers which are complementary to the known sequence (Tn5) and the arbitrary degenerate primers which could be complementary to the opposite unknown site. The specific primers (SP) are PR1, PR2, PR3, PF1, PF2, PF3, and TF1-2S1 to TF1-2A6 primers for opposite direction (Additional file 1, Table S1). In addition, the arbitrary degenerate primers (AD) N1, N2, and N3 were respectively used as simultaneous PCR amplification (see above).

\section{Acknowledgements}

The support of this work by grants from the National Science Council (grants NSC-97-2313-B-005-027-MY3) of Taiwan (R.O.C.) is gratefully acknowledged.

\section{Author details}

'Department of Chemistry, National Chung-Hsing University, 250, Kuokuang Rd., Taichung, 402, Taiwan. 'Division of Pulmonary Medicine, Department of 
Internal Medicine, Chang Gung Memorial Hospital, Keelung, 204, Taiwan. ${ }^{3}$ Department of plant pathology, National Chung-Hsing University, 250, Kuokuang Rd., Taichung, 402, Taiwan.

\section{Authors' contributions}

YC participated in the discovery and characterization of Carocin S2, and he wrote this manuscript. $J$ participated in protein purification. HP participated in manuscript preparation. KC supported the PCC strain SP33 and for insightful discussion and guidance. DY conceived of the study, participated in its design, and corrected the manuscript. All authors read and approved the final version of the manuscript.

\section{Received: 21 September 2010 Accepted: 12 May 2011}

Published: 12 May 2011

\section{References}

1. Pe'rombelon MCM: Potato diseases caused by soft-rot erwinias: an overview of pathogenesis. The role of pectic enzymes in plant pathogenesis. Plant Pathol 2002, 51:1-12.

2. Collmer A, Keen NT: The role of pectic enzymes in plant pathogenesis. Annu Rev Phytopathol 1986, 24:383-409.

3. Barras F, Van Gijsegem F, Chatterjee AK: Extracellular enzymes and pathogenesis of soft-rot Erwinia. Annu Rev Phytopathol 1994, 32:201-234.

4. Eckert JW, Ogawa JM: The Chemical Control of Postharvest Diseases: Deciduous Fruits, Berries, Vegetables and Root/Tuber Crops. Annu Rev Phytopathol 1988, 26:433-469.

5. Kikumoto T, Kyeremeh AG, Chuang DY, Gunji Y, Takahara Y, Ehara Y: Biological Control of Soft Rot of Chinese Cabbage Using Single and Mixed Treatments of Bacteriocin-producing Avirulent Mutants of Erwinia carotovora subsp. carotovora. J Gen Plant Pathol 2000, 66:264-268.

6. Jack RW, Tagg JR, Ray B: Bacteriocins of Gram-Positive Bacteria. Microbiol Rev 1995, 59:171-200.

7. Daw MA, Falkiner FR: Bacteriocins: Nature, Function and Structure. Micron 1996, 27:467-479.

8. Cascales E, Buchanan SK, Duche D, Kleanthous C, Lloube's R, Postle K Riley M, Slatin S, Cavard D: Colicin Biology. Microbiol Mol Biol Rev 2007, 71:158-229.

9. Boon T: Inactivation of Ribosomes In Vitro by Colicin E3. Proc Natl Acad Sci USA 1971, 68:2421-2425.

10. Mosbahi K, Walker D, James R, Moore GR, Kleanthous C: Global structural rearrangement of the cell penetrating ribonuclease colicin $E 3$ on interaction with phospholipid membranes. Protein Sci 2006, 15:620-627.

11. Senior BW, Holland IB: Effect of colicin E3 upon the 30S ribosomal subunit of Escherichia coli. Proc Natl Acad Sci USA 1971, 68:959-963.

12. Zarivach R, Ben-Zeev E, Wu N, Auerbach T, Bashan A, Jakes K, Dickman K, Kosmidis A, Schluenzen F, Yonath A, Eisenstein M, Shoham M: On the interaction of colicin E3 with the ribosome. Biochimie 2002, 84:447-454.

13. Lancaster LE, Savelsbergh A, Kleanthous C, Wintermeyer W, Rodnina MV: Colicin E3 cleavage of 16S rRNA impairs decoding and accelerates tRNA translocation on Escherichia coli ribosomes. Mol Microbiol 2008, 69:390-401.

14. Soelaiman S, Jakes K, Wu N, Li C, Shoham M: Crystal structure of colicin E3: implications for cell entry and ribosome inactivation. Mol Cell 2001, 8:1053-1062

15. Jakes KS, Zinder ND: Highly purified colicin E3 contains immunity protein. Proc Natl Acad Sci USA 1974, 71:3380-3384.

16. Jakes K, Zinder ND, Boon T: Purification and properties of colicin E3 immunity protein. J Biol Chem 1974, 249:438-444.

17. Vankemmelbeke M, Zhang Y, Moore GR, Kleanthous C, Penfold CN, James R: Energy-dependent immunity protein release during toldependent nuclease colicin translocation. J Biol Chem 2009, 284:18932-18941.

18. Kageyama M, Kobayashi M, Sano Y, Masaki H: Construction and characterization of pyocin-colicin chimeric proteins. J Bacterio/ 1996, 178:103-110.

19. Ogawa T, Tomita K, Ueda T, Watanabe K, Uozumi T, Masaki H: A cytotoxic ribonuclease targeting specific transfer RNA anticodons. Science 1999, 283:2097-2100.

20. Tomita K, Ogawa T, Uozumi T, Watanabe K, Masaki H: A cytotoxic ribonuclease which specifically cleaves four isoaccepting arginine tRNAs at their anticodon loops. Proc Natl Acad Sci USA 2000, 97:8278-8283.
21. de Zamaroczy M, Mora L, Lecuyer A, Géli V, Buckingham RH: Cleavage of Colicin D Is Necessary for Cell Killing and Requires the Inner Membrane Peptidase LepB. Mol Cell 2001, 8:159-168.

22. Nguyen AH, Tomita T, Hirota M, Sato T, Kamio $Y$ : A simple purification method and morphology and component analyses for carotovoricin Er, a phage-tail-like bacteriocin from the plant pathogen Erwinia carotovora Er. Biosci Biotechnol Biochem 1999, 63:1360-1369.

23. Chuang DY, Chien YC, Wu HP: Cloning and Expression of the Erwinia carotovora subsp. carotovora Gene Encoding the Low-Molecular-Weight Bacteriocin Carocin S1. J Bacteriol 2007, 189:620-626.

24. Chan YC, Wu HP, Chuang DY: Extracellular secretion of Carocin S1 in Pectobacterium carotovorum subsp. carotovorum occurs via the type III secretion system integral to the bacterial flagellum. BMC Microbiol 2009, 9:181.

25. Bradley DE: Ultrastructure of bacteriophage and bacteriocins. Bacterio/ Rev 1967, 31:230-314.

26. Ross W, Gosink KK, Salomon J, Igarashi K, Zou C, Ishihama A, Severinov K, Gourse RL: A third recognition element in bacterial promoters: DNA binding by the alpha subunit of RNA polymerase. Science 1993, 262:1407-1413.

27. Sharma O, Cramer WA: Minimum length requirement of the flexible Nterminal translocation subdomain of colicin E3. J Bacteriol 2007, 189:363-368.

28. Roh E, Park TH, Kim Ml, Lee S, Ryu S, Oh CS, Rhee S, Kim DH, Park BS, Heu S: Characterization of a new bacteriocin, Carocin D, from Pectobacterium carotovorum subsp. carotovorum Pcc21. Appl Environ Microbiol 2010, 76:7541-7549.

29. Chavan M, Rafi H, Wertz J, Goldstone C, Riley MA: Phage associated bacteriocins reveal a novel mechanism for bacteriocin diversification in Klebsiella. J Mol Evol 2005, 60:546-556.

30. de Zamaroczy M, Buckingham $\mathrm{RH}$ : Importation of nuclease colicins into $E$ coli cells: endoproteolytic cleavage and its prevention by the immunity protein. Biochimie 2002, 84:423-432.

31. Mora L, Klepsch M, Buckingham RH, Heurgué-Hamard V, Kervestin S, de Zamaroczy M: Dual roles of the central domain of colicin D tRNase in TonB-mediated import and in immunity. J Biol Chem 2008, 283:4993-5003

32. Hirao I, Harada Y, Nojima T, Osawa Y, Masaki H, Yokoyama S: In vitro selection of RNA aptamers that bind to colicin E3 and structurally resemble the decoding site of $16 \mathrm{~S}$ ribosomal RNA. Biochemistry 2004, 43:3214-3221.

33. Ohno S, Imahori K: Colicin E3 is an endonuclease. J Biochem 1978, 84:1637-1640.

34. Sano Y, Kobayashi M, Kageyama M: Functional domains of S-type pyocins deduced from chimeric molecules. J Bacteriol 1993, 175:6179-6185.

35. Fredericq P: Colicins. Annu Rev Microbiol 1957, 11:7-22.

36. Sambrook J, Fritsch EF, Maniatis T: Molecular cloning: a laboratory manual. 2 edition. Cold Spring Harbor Laboratory Press, Cold Spring Harbor, NY; 1989.

37. Liu YG, Whittier RF: Thermal asymmetric interlaced PCR: automatable amplification and sequencing of insert end fragments from P1 and YAC clones for chromosome walking. Genomics 1995, 25:674-681.

38. Metzger M, Bellemann P, Schwartz T, Geider K: Site-directed and transposon-mediated mutagenesis with pfd-plasmids by electroporation of Erwinia amylovora and Escherichia coli cells. Nucleic Acids Res 1992, 20:2265-2270.

39. Hanahan D: Studies on transformation of Escherichia coli with plasmids. $J$ Mol Biol 1983, 166:557-580.

40. Liu H, Naismith $\mathrm{JH}$ : An efficient one-step site-directed deletion, insertion, single and multiple-site plasmid mutagenesis protocol. BMC Biotechnol 2008, 8:91.

41. Garinot-Schneider C, Pommer AJ, Moore GR, Kleanthous C, James R: Identification of putative active-site residues in the DNase domain of colicin E9 by random mutagenesis. J Mol Biol 1996, 260:731-742.

42. Silberklang M, Gillum AM, RajBhandary UL: The use of nuclease P1 in sequence analysis of end group labeled RNA. Nucleic Acids Res 1977 4:4091-4108

43. Bruce AG, Uhlenbeck OC: Reactions at the termini of tRNA with T4 RNA ligase. Nucleic Acids Res 1978, 5:3665-77.

44. Gantotti BV, Kindle KL, Beer SV: Transfer of the drug-resistance transposon Tn5 to Erwinia herbicola and the induction of the insertion Mutation. Curr Microbiol 1981, 6:417-425. 
45. Wood WB: Host specificity of DNA produced by Escherichia coli: bacterial mutations affecting the restriction and modification of DNA. J Mol Biol 1966, 16:118-133.

46. Nakano $Y$, Yoshida $Y$, Yamashita $Y$, Koga T: Construction of a series of pACYC-derived plasmid vectors. Gene 1995, 162:157-158.

doi:10.1186/1471-2180-11-99

Cite this article as: Chan et al:: Cloning, purification, and functional

characterization of Carocin S2, a ribonuclease bacteriocin produced by Pectobacterium carotovorum. BMC Microbiology 2011 11:99.

Submit your next manuscript to BioMed Central and take full advantage of:

- Convenient online submission

- Thorough peer review

- No space constraints or color figure charges

- Immediate publication on acceptance

- Inclusion in PubMed, CAS, Scopus and Google Scholar

- Research which is freely available for redistribution

Submit your manuscript at www.biomedcentral.com/submit 